\title{
WASP-20b and WASP-28b: a hot Saturn and a hot Jupiter in near-aligned orbits around solar-type stars ${ }^{\star} \star \star \star$
}

D. R. Anderson ${ }^{1}$, A. Collier Cameron ${ }^{2}$, C. Hellier ${ }^{1}$, M. Lendl ${ }^{3}$, T. A. Lister ${ }^{4}$, P. F. L. Maxted ${ }^{1}$, D. Queloz ${ }^{3,5}$, B. Smalley ${ }^{1}$, A. M. S. Smith ${ }^{1,6}$, A. H. M. J. Triaud ${ }^{3,7}$, D. J. A. Brown ${ }^{8,9}$, M. Gillon ${ }^{10}$, M. Neveu-VanMalle ${ }^{3}$, F. Pepe ${ }^{3}$, D. Pollacco ${ }^{8}$, D. Ségransan ${ }^{3}$, S. Udry ${ }^{3}$, R. G. West ${ }^{8}$, and P. J. Wheatley ${ }^{8}$

\author{
1 Astrophysics Group, Keele University, Staffordshire ST5 5BG, UK \\ e-mail: d.r.anderson@keele.ac.uk \\ 2 SUPA, School of Physics and Astronomy, University of St. Andrews, North Haugh, Fife KY16 9SS, UK \\ 3 Observatoire de Genève, Université de Genève, 51 Chemin des Maillettes, 1290 Sauverny, Switzerland \\ ${ }^{4}$ Las Cumbres Observatory Global Telescope Network, 6740 Cortona Dr. Suite 102, Goleta, CA 93117, USA \\ Cavendish Laboratory, J J Thomson Avenue, Cambridge, CB3 0HE, UK \\ ${ }^{6}$ N. Copernicus Astronomical Centre, Polish Academy of Sciences, Bartycka 18, 00-716 Warsaw, Poland \\ 7 Department of Physics, and Kavli Institute for Astrophysics and Space Research, MIT, Cambridge, MA 02139, USA \\ 8 Department of Physics, University of Warwick, Coventry CV4 7AL, UK \\ 9 Astrophysics Research Centre, School of Mathematics \& Physics, Queen's University, University Road, Belfast BT7 1NN, UK \\ 10 Institut d'Astrophysique et de Géophysique, Université de Liège, Allée du 6 Août, 17, Bât. B5C, Liège 1, Belgium
}

Received 6 February 2014 / Accepted 5 December 2014

\section{ABSTRACT}

\begin{abstract}
We report the discovery of the planets WASP-20b and WASP-28b along with measurements of their sky-projected orbital obliquities. WASP-20b is an inflated, Saturn-mass planet $\left(0.31 M_{\text {Jup }} ; 1.46 R_{\text {Jup }}\right)$ in a 4.9-day, near-aligned $\left(\lambda=12.7 \pm 4.2^{\circ}\right)$ orbit around CD-24 102 $(V=10.7$; F9). Due to the low density of the planet and the apparent brightness of the host star, WASP-20 is a good target for atmospheric characterisation via transmission spectroscopy. WASP-28b is an inflated, Jupiter-mass planet $\left(0.91 M_{\text {Jup }} ; 1.21 R_{\text {Jup }}\right)$ in a 3.4-day, near-aligned $\left(\lambda=8 \pm 18^{\circ}\right)$ orbit around a $V=12$, F8 star. As intermediate-mass planets in short orbits around aged, cool stars $\left(7_{-1}^{+2}\right.$ Gyr and $6000 \pm 100 \mathrm{~K}$ for WASP-20; $5_{-2}^{+3}$ Gyr and $6100 \pm 150 \mathrm{~K}$ for WASP-28), their orbital alignment is consistent with the hypothesis that close-in giant planets are scattered into eccentric orbits with random alignments, which are then circularised and aligned with their stars' spins via tidal dissipation.
\end{abstract}

Key words. planetary systems - stars: individual: WASP-20b - stars: individual: WASP-28b

\section{Introduction}

Planets that transit relatively bright host stars $(V<13)$ are proving a rich source of information for the nascent field of exoplanetology. To date, the main discoverers of these systems are the ground-based transit surveys HATNet and SuperWASP and the space mission Kepler (Bakos et al. 2004; Pollacco et al. 2006; Borucki et al. 2010).

One parameter that, uniquely, we can determine for transiting planets is obliquity $(\Psi)$, the angle between a star's rotation axis and a planet's orbital axis. We do this by taking spectra of a star during transit: as the planet obscures a portion of the rotating star it causes a distortion of the observed stellar line profile, which manifests as an anomalous radial-velocity (RV) signature

^ Based on observations made with: the WASP-South (South Africa) and SuperWASP-North (La Palma) photometric survey instruments; the $\mathrm{C} 2$ and EulerCam cameras and the CORALIE spectrograph, all mounted on the 1.2-m Euler-Swiss telescope (La Silla); the HARPS spectrograph on the ESO 3.6-m telescope (La Silla) under programs 072.C-0488, 082.C-0608, 084.C-0185, and 085.C-0393; and LCOGT's Faulkes Telescope North (Maui) and Faulkes Telescope South (Siding Spring).

$\star \star$ Full Tables 2 and 3 are only available at the CDS via anonymous ftp to cdsarc.u-strasbg. fr $(130.79 .128 .5)$ or via

http://cdsarc.u-strasbg.fr/viz-bin/qcat?]/A+A/575/A61 known as the Rossiter-McLaughlin (RM) effect (Rossiter 1924; McLaughlin 1924). The shape of the RM effect is sensitive to the path a planet takes across the disc of a star relative to the stellar spin axis. If we have a constraint on the inclination of the stellar spin axis relative to the sky plane $\left(I_{*}\right)$, then we can determine the true obliquity $(\Psi)$, otherwise we can only determine the sky-projected obliquity $(\lambda)$. The two are related by:

$\cos \Psi=\cos I_{*} \cos i_{\mathrm{P}}+\sin I_{*} \sin i_{\mathrm{P}} \cos \lambda$

where $i_{\mathrm{P}}$ is the inclination of the orbital axis to the sky plane.

The obliquity of a short-period, giant planet may be indicative of the manner in which it arrived in its current orbit from farther out, where it presumably formed. As the angular momenta of a star and its planet-forming disc both derive from that of their parent molecular cloud, stellar spin and planetary orbital axes are expected to be, at least initially, aligned. Migration via tidal interaction with the gas disc is expected to preserve initial spin-orbit alignment (Lin et al. 1996; Marzari \& Nelson 2009), but almost half (33 of 74) of the orbits so far measured are misaligned and approximately 10 of those are retrograde ${ }^{1}$.

\footnotetext{
René Heller maintains a list of measurements and references at http://www.physics.mcmaster.ca/ rheller/content/ main_HRM.html
} 
Table 1. Summary of observations.

\begin{tabular}{|c|c|c|c|c|c|}
\hline Facility & Date & $N_{\text {obs }}$ & $\begin{array}{l}T_{\exp } \\
(\mathrm{s})\end{array}$ & Filter & Issue \\
\hline \multicolumn{6}{|l|}{ WASP-20: } \\
\hline WASP-South & 2006 May-2007 Nov. & 9600 & 30 & Broad $(400-700 \mathrm{~nm})$ & - \\
\hline Euler/CORALIE & 2008 Jul.-2013 Oct. & 56 & 1800 & Spectroscopy & - \\
\hline ESO-3.6 m/HARPS & 2008 Aug.-2011 Sep. & 20 & $600-1800$ & Spectroscopy & - \\
\hline FTS/Spectral & 2008 Oct. 25 & 151 & 40 & $z^{\prime}$ & autoguider \\
\hline ESO-3.6 m/HARPS & 2009 Oct. 22 & 43 & $300-400 / 1200$ & Spectroscopy & - \\
\hline FTN/Merope & 2011 Aug. 20 & 70 & 70 & $z^{\prime}$ & rotator \\
\hline Euler/EulerCam & 2011 Aug. 29 & 237 & 20 & Gunn-r & cloud \\
\hline Euler/EulerCam & 2011 Sep. 03 & 156 & 70 & Gunn- $r$ & - \\
\hline $\begin{array}{l}\text { Euler/EulerCam } \\
\text { WASP-28 }\end{array}$ & 2011 Sep. 08 & 201 & 30 & Gunn- $r$ & - \\
\hline WASP-South & 2008 Jun.-2009 Nov. & 10100 & 30 & Broad $(400-700 \mathrm{~nm})$ & - \\
\hline SuperWASP-North & 2008 Aug.-2010 Sep. & 6600 & 30 & Broad $(400-700 \mathrm{~nm})$ & - \\
\hline Euler/CORALIE & 2009 Jun.-2012 Dec. & 26 & 1800 & Spectroscopy & - \\
\hline FTN/Merope & 2009 Oct. 21 & 227 & 40 & $z^{\prime}$ & unknown \\
\hline ESO-3.6 m/HARPS & 2010 Aug. 17-19 & 33 & $600 / 1200$ & Spectroscopy & - \\
\hline Euler/C2 & 2010 Sep. 03 & 217 & 25 & Gunn- $r$ & - \\
\hline
\end{tabular}

These results are consistent with the hypothesis that some or all close-in giant planets arrive in their orbits by planet-planet and/or star-planet scattering, which can drive planets into eccentric, misaligned orbits, and tidal friction, which circularises, shortens and aligns orbits (see the following empirical-based papers: Triaud et al. 2010; Winn et al. 2010; Naoz et al. 2011; Albrecht et al. 2012; also see the following model-based papers: Fabrycky \& Tremaine 2007; Nagasawa et al. 2008; Matsumura et al. 2010; Naoz et al. 2011).

Systems with short tidal timescales (those with short scaled orbital major semi-axes, $a / R_{*}$, and high planet-to-star mass ratios) tend to be aligned (Albrecht et al. 2012, and references therein). A broad range of obliquities is observed for stars with $T_{\text {eff }}>6250 \mathrm{~K}$, for which tidal realignment processes may be inefficient due to the absence of a substantial convective envelope (Winn et al. 2010; Schlaufman 2010). Limiting focus to stars more massive than $1.2 M_{\odot}$, for which age determinations are more reliable, Triaud (2011) noted that systems older than 2.5 Gyr tend to be aligned; this could be indicative of the time required for orbital alignment or of the timescale over which hotter stars develop a substantial convective envelope as they evolve.

A major hurdle to overcome for any hypothesis involving realignment is tidal dissipation seems to cause both orbital decay and realignment on similar timescales (Barker \& Ogilvie 2009). Alternative hypotheses suggest that misalignments arise via reorientations of either the disc or the stellar spin and that migration then proceeds via planet-disc interactions (e.g. Bate et al. 2010; Lai et al. 2011; Rogers et al. 2012). However, observations of discs well-aligned with their stellar equators suggest that tilting of the star or the disc rarely occurs (Watson et al. 2011; Greaves et al. 2014).

Here we present the discovery and obliquity determinations of the planets transiting the stars WASP-20 (CD-24 102) and WASP-28 (2MASS J23342787-0134482), and we interpret the results under the hypothesis of migration via scattering and tidal dissipation.

\section{Observations}

We provide a summary of observations in Table 1. The WASP (Wide Angle Search for Planets) photometric survey (Pollacco et al. 2006) monitors bright stars $(V=9.5-13.5)$ using two eight-camera arrays, each with a field of view of $450 \mathrm{deg}^{2}$. Each array observes up to eight pointings per night with a cadence of 5-10 min, and each pointing is followed for around five months per season. The WASP-South station (Hellier et al. 2011) is hosted by the South African Astronomical Observatory and the SuperWASP-North station (Faedi et al. 2011) is hosted by the Isaac Newton Group at the Observatorio del Roque de Los Muchachos on La Palma. The WASP data were processed and searched for transit signals as described in Collier Cameron et al. (2006) and the candidate selection process was performed as described in Collier Cameron et al. (2007). We observed periodic dimmings in the WASP lightcurves of WASP-20 and WASP-28 with periods of $4.8996 \mathrm{~d}$ and $3.4088 \mathrm{~d}$, respectively (see the top panels of Figs. 1 and 2). We searched the WASP lightcurves for modulation as could be caused by magnetic activity and stellar rotation (Maxted et al. 2011). We did not detect any significant, repeated signals and we find that any modulation must be below the 2-mmag level.

We obtained 56 spectra of WASP-20 and 26 spectra of WASP-28 with the CORALIE spectrograph mounted on the Euler-Swiss 1.2-m telescope (Baranne et al. 1996; Queloz et al. 2000). We obtained a further 63 spectra of WASP-20 with the HARPS spectrograph mounted on the 3.6-m ESO telescope (Mayor et al. 2003), including a sequence of 43 spectra taken through the transit of the night of 2009 October 22. We obtained a further 33 spectra of WASP-28 with HARPS, including 30 spectra taken through the transit of the night of 2010 August 17. Radial-velocity measurements were computed by weighted cross-correlation (Baranne et al. 1996; Pepe et al. 2005 ) with a numerical G2-spectral template (Table 2). We detected RV variations with periods similar to those found from the WASP photometry and with semi-amplitudes consistent with planetary-mass companions. We plot the RVs, phased on the transit ephemerides, in the third panel of Figs. 1 and 2 and we highlight the RM effects in Fig. 3.

For each star, we tested the hypothesis that the RV variations are due to spectral-line distortions caused by a blended eclipsing binary or starspots by performing a line-bisector analysis of the cross-correlation functions (Queloz et al. 2001). The lack of any significant correlation between bisector span and RV supports our conclusion that the periodic dimming and RV variation of each system are caused by a transiting planet (Fig. 4). This is further supported by our observation of the RM effect of each 

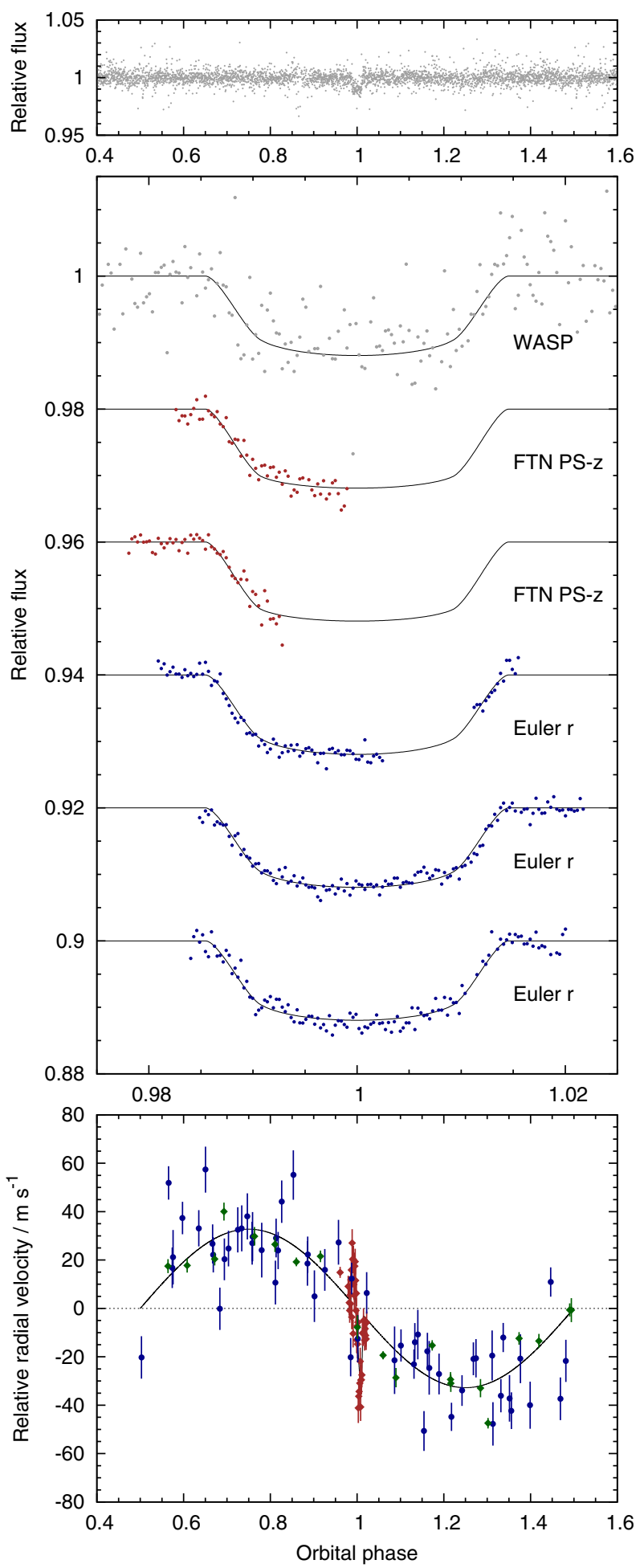

Fig. 1. WASP-20b discovery data. Top panel: WASP lightcurve folded on the transit ephemeris. Middle panel: transit lightcurves from facilities as labelled, offset for clarity and binned with a bin width of two minutes. The best-fitting transit model is superimposed. Bottom panel: the radial velocities (CORALIE in blue, HARPS in green and HARPS covering the transit in brown) with the best-fitting circular Keplerian orbit model and the RM effect model.

system: the $v \sin I_{*}$ values from our fits to the RM effects are consistent with the values we obtain from spectral line broadening (see Sects. 3 and 4).

We performed follow-up transit observations to refine the systems' parameters using the Euler-Swiss 1.2-m telescope
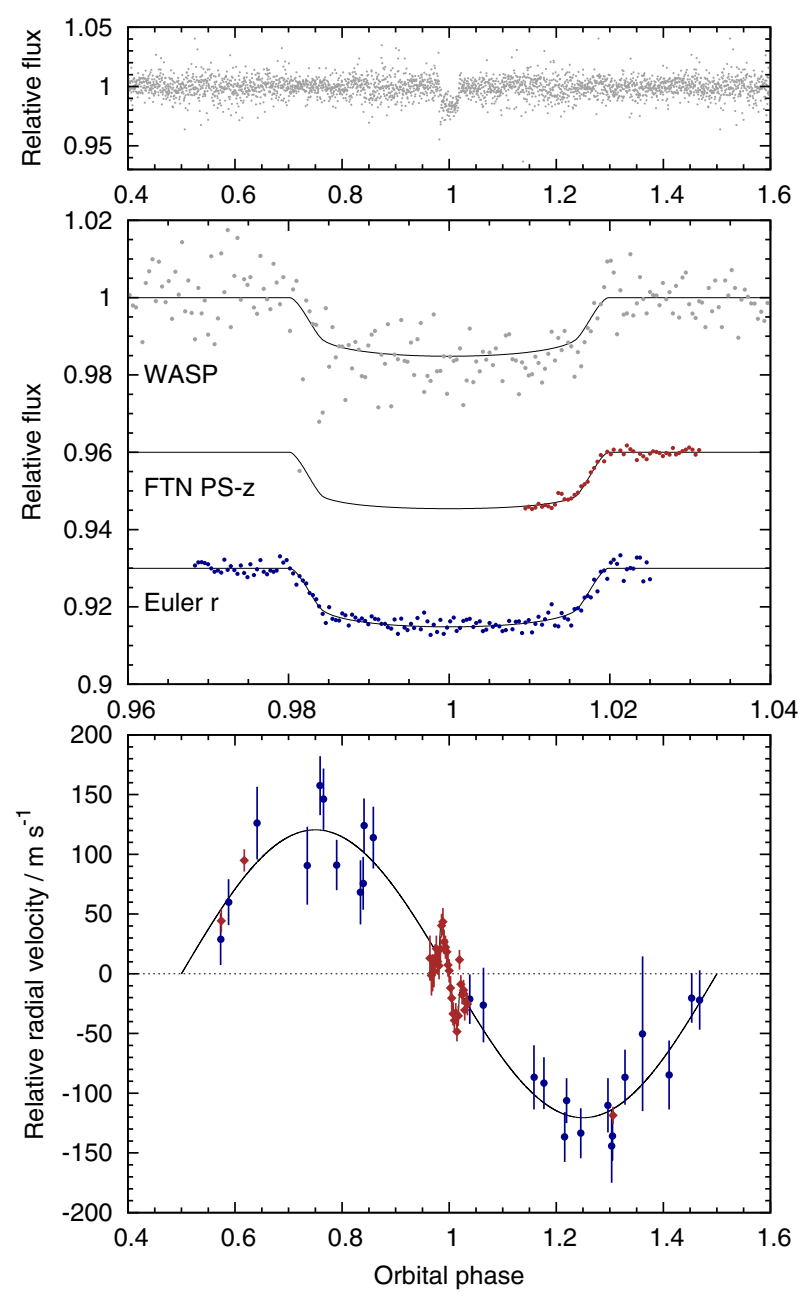

Fig. 2. WASP-28b discovery data. Caption as for Fig. 1.

Table 2. Radial-velocity measurements.

\begin{tabular}{lllrrr}
\hline \hline Star & Spect. & $\begin{array}{l}\text { BJD(UTC) } \\
-2450000 \\
(\text { day })\end{array}$ & RV & \multicolumn{1}{l}{$\sigma_{\mathrm{RV}}$} & BS \\
& & $\left.\mathrm{km} \mathrm{s}^{-1}\right)$ & $\left(\mathrm{km} \mathrm{s}^{-1}\right)$ & $\left(\mathrm{km} \mathrm{s}^{-1}\right)$ \\
\hline W-20 & COR & 4655.88265 & 1.3411 & 0.0074 & 0.0613 \\
W-20 & COR & 4658.86065 & 1.2969 & 0.0103 & 0.0490 \\
$\ldots$ & & & & & \\
W-28 & HAR & 5428.71664 & 24.2674 & 0.0098 & 0.0021 \\
W-28 & HAR & 5428.86297 & 24.3180 & 0.0094 & 0.0145 \\
\hline
\end{tabular}

Notes. The uncertainties are the formal errors (i.e. with no added jitter). The uncertainties on the bisector spans (BS) are $2 \sigma_{\mathrm{RV}}$. This table is available in its entirety via the CDS.

(Lendl et al. 2012), and LCOGT's 2.0-m Faulkes Telescopes North and South (Brown et al. 2013) (see the middle panels of Figs. 1 and 2). Some transit lightcurves were affected by technical problems or poor weather, as indicated in Table 1.

\section{Stellar parameters from spectra}

The 9 individual HARPS spectra from 2008 of WASP-20 were co-added and a total of 18 individual CORALIE spectra of WASP-28 were co-added to produce one spectrum per star, with typical signal-to-noise ratio of 150:1 (WASP-20) and 70:1 (WASP-28). The standard pipeline reduction products were used in the analysis. 

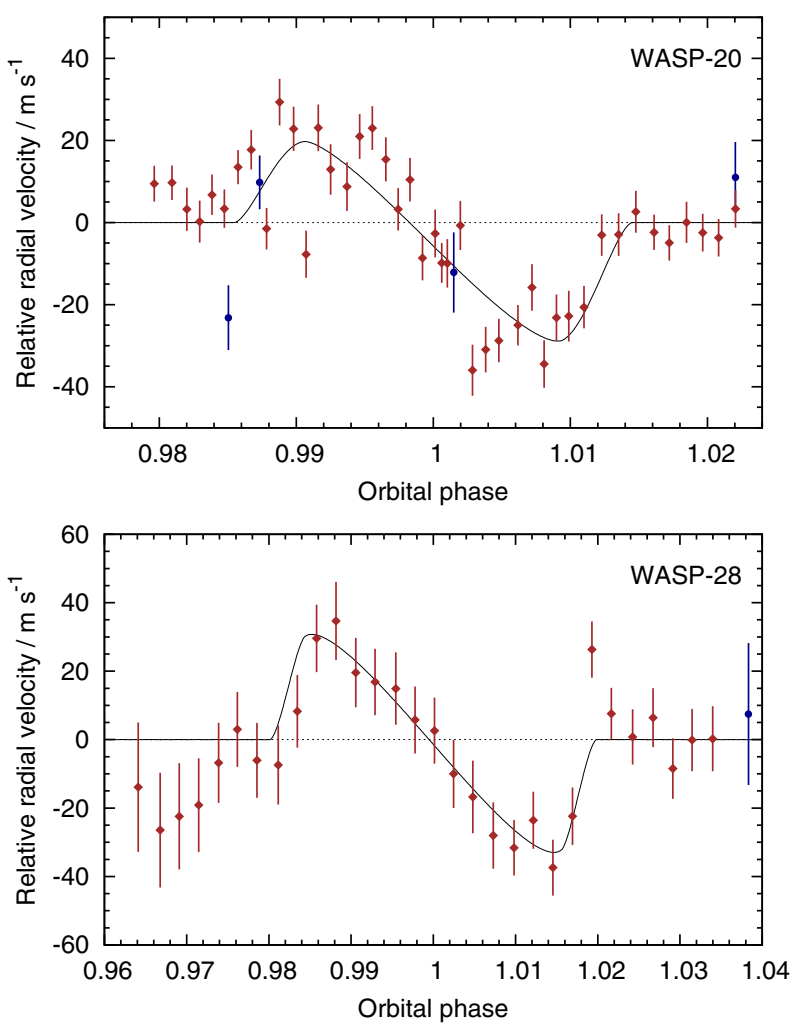

Fig. 3. Rossiter-McLaughlin effects, or spectroscopic transits, of WASP-20b and WASP-28b. The CORALIE RVs are shown in blue and the HARPS RVs are shown in brown. The Keplerian orbits have been subtracted. For each planet, the observed apparent redshift, followed by an apparent blueshift of similar amplitude, indicate the orbit to be prograde and, probably, aligned with the stellar rotation. The alignment of the orbit of WASP-28b is less certain due to its low impact parameter. The lower precision of the earlier WASP-28 RVs resulted because the sequence started at an airmass of 1.7.

Table 3. Follow-up photometry.

\begin{tabular}{llllll}
\hline \hline Set & Star & Tel. & $\begin{array}{l}\text { BJD(UTC) } \\
-2450000 \\
\text { (day) }\end{array}$ & Flux, $F$ & $\sigma_{F}$ \\
\hline 1 & W-20 & FTN & 4765.04209 & 0.99958 & 0.00179 \\
1 & W-20 & FTN & 4765.04283 & 1.00022 & 0.00163 \\
$\ldots$ & & & & & \\
7 & W-28 & Euler & 5443.88762 & 0.9971 & 0.0027 \\
7 & W-28 & Euler & 5443.88852 & 1.0006 & 0.0027 \\
\hline
\end{tabular}

Notes. The flux values are differential and normalised to the out-oftransit levels. The uncertainties are the formal errors (i.e. they have not been rescaled). This table is available in its entirety via the CDS.

The analysis was performed using the methods given in Gillon et al. (2009). The $\mathrm{H}_{\alpha}$ line was used to determine the effective temperature $\left(T_{\text {eff }}\right)$, while the $\mathrm{Na} \mathrm{I} \mathrm{D} \mathrm{and} \mathrm{Mg}$ I b lines were used as surface gravity $\left(\log g_{*}\right)$ diagnostics. The parameters obtained from the analysis are listed in Table 4. The elemental abundances were determined from equivalent width measurements of several clean and unblended lines. A value for microturbulence $\left(\xi_{\mathrm{t}}\right)$ was determined from Fe I using the method of Magain (1984). The quoted error estimates include that given by the uncertainties in $T_{\text {eff }}, \log g_{*}$ and $\xi_{\mathrm{t}}$, as well as the scatter due to measurement and atomic data uncertainties.

We assumed values for macroturbulence $\left(v_{\text {mac }}\right)$ of $4.5 \pm$ $0.3 \mathrm{~km} \mathrm{~s}^{-1}$ and $4.7 \pm 0.3 \mathrm{~km} \mathrm{~s}^{-1}$ for WASP-20 and WASP-28,
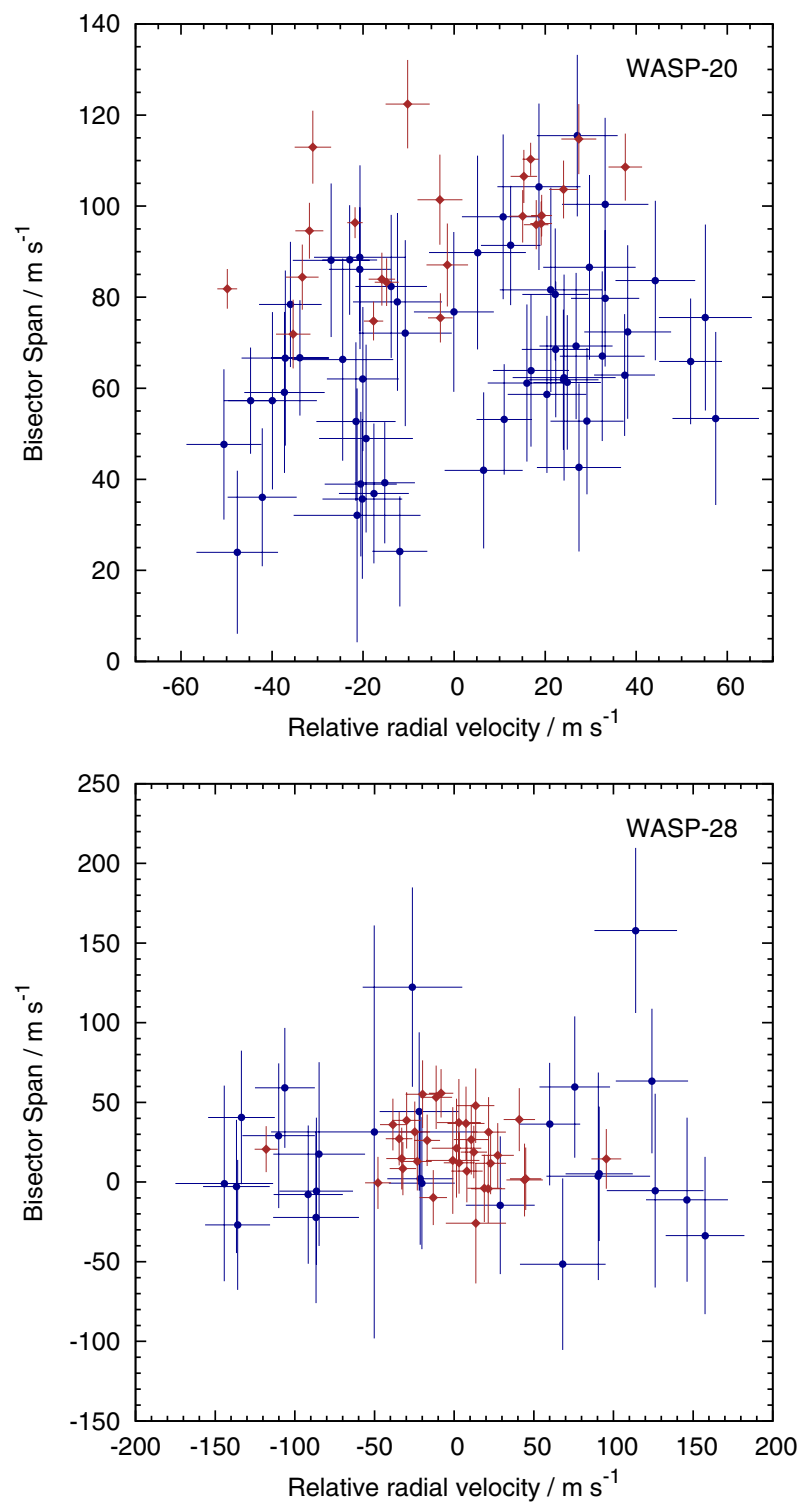

Fig. 4. The lack of any significant correlation between bisector span and radial velocity excludes transit mimics and supports our conclusion that each system contains a transiting planet.

respectively. These were based on the tabulation by Gray (2008) and the instrumental FWHMs of $0.065 \AA$ (HARPS) and $0.11 \pm 0.01 \AA$ (CORALIE), as determined from the telluric lines around $6300 \AA$. We determined the projected stellar rotation velocity $\left(v \sin I_{*}\right)$ by fitting the profiles of several unblended Fe I lines. We determined the $v \sin I_{*}$ of WASP-20 from the 62 HARPS spectra obtained up to 2010 and the $v \sin I_{*}$ of WASP-28 from all 33 available HARPS spectra.

\section{System parameters from the RV and transit data}

We determined the parameters of each system from a simultaneous fit to the lightcurve and RV data. The fit was performed using the current version of the Markov-chain Monte Carlo (MCMC) code described by Collier Cameron et al. (2007) and Pollacco et al. (2008).

The transit lightcurves are modelled using the formulation of Mandel \& Agol (2002) with the assumption that the planet is much smaller than the star. Limb-darkening was accounted for 
Table 4. Stellar parameters from the spectra.

\begin{tabular}{|c|c|c|}
\hline Parameter & WASP-20 & WASP-28 \\
\hline Star & CD-24 102 & 2MASS J23342787-0134482 \\
\hline Constellation & Cetus & Pisces \\
\hline RA (J2000) & $00^{\mathrm{h}} 20^{\mathrm{m}} 38^{\mathrm{s}} .53$ & $23^{\mathrm{h}} 34^{\mathrm{m}} 27.87$ \\
\hline Dec (J2000) & $-23^{\circ} 56^{\prime} 08^{\prime \prime} .6$ & $-01^{\circ} 34^{\prime} 48^{\prime \prime} .2$ \\
\hline$B$ & 11.17 & 12.50 \\
\hline V & 10.68 & 12.03 \\
\hline$J$ & 9.70 & 11.08 \\
\hline$H$ & 9.42 & 10.76 \\
\hline$K$ & 9.39 & 10.73 \\
\hline$T_{\text {eff }}$ & $6000 \pm 100 \mathrm{~K}$ & $6100 \pm 150 \mathrm{~K}$ \\
\hline $\log g_{*}$ & $4.40 \pm 0.15$ & $4.5 \pm 0.2$ \\
\hline$\xi_{\mathrm{t}}$ & $1.2 \pm 0.1 \mathrm{~km} \mathrm{~s}^{-1}$ & $1.2 \pm 0.1 \mathrm{~km} \mathrm{~s}^{-1}$ \\
\hline$v \sin I_{*}$ & $3.5 \pm 0.5 \mathrm{~km} \mathrm{~s}^{-1}$ & $3.1 \pm 0.6 \mathrm{~km} \mathrm{~s}^{-1}$ \\
\hline$[\mathrm{Fe} / \mathrm{H}]$ & $-0.01 \pm 0.06$ & $-0.29 \pm 0.10$ \\
\hline$[\mathrm{Si} / \mathrm{H}]$ & $+0.03 \pm 0.09$ & $-0.22 \pm 0.10$ \\
\hline$[\mathrm{Ca} / \mathrm{H}]$ & $+0.09 \pm 0.11$ & $-0.20 \pm 0.12$ \\
\hline$[\mathrm{Sc} / \mathrm{H}]$ & $+0.03 \pm 0.06$ & $\cdots$ \\
\hline$[\mathrm{Ti} / \mathrm{H}]$ & $+0.09 \pm 0.11$ & $-0.21 \pm 0.07$ \\
\hline$[\mathrm{V} / \mathrm{H}]$ & $+0.09 \pm 0.07$ & $\ldots$ \\
\hline$[\mathrm{Cr} / \mathrm{H}]$ & $-0.04 \pm 0.06$ & $\ldots$ \\
\hline$[\mathrm{Mn} / \mathrm{H}]$ & $-0.01 \pm 0.08$ & .. \\
\hline$[\mathrm{Co} / \mathrm{H}]$ & $-0.02 \pm 0.08$ & $\ldots$ \\
\hline$[\mathrm{Ni} / \mathrm{H}]$ & $0.00 \pm 0.06$ & $-0.28 \pm 0.08$ \\
\hline $\log \mathrm{A}(\mathrm{Li})$ & $2.40 \pm 0.10$ & $2.52 \pm 0.12$ \\
\hline Spectral type & F9 & F8 \\
\hline Age & $7_{-1}^{+2} \mathrm{Gyr}$ & $5_{-2}^{+3} \mathrm{Gyr}$ \\
\hline Distance & $210 \pm 20 \mathrm{pc}$ & $410 \pm 70 \mathrm{pc}$ \\
\hline
\end{tabular}

Notes. We determined $v \sin I_{*}$ from different subsets of spectra than the other parameters, as described in the text. The magnitudes are from the NOMAD catalogue (Zacharias et al. 2004). The spectral types are estimated from $T_{\text {eff }}$ using Table B.1 in Gray (2008).

using a four-coefficient, nonlinear limb-darkening model, using coefficients appropriate to the passbands from the tabulations of Claret $(2000,2004)$. The coefficients are interpolated once using the values of $\log g_{*}$ and $[\mathrm{Fe} / \mathrm{H}]$ in Table 4, but are interpolated at each MCMC step using the latest value of $T_{\text {eff }}$. The coefficient values corresponding to the best-fitting value of $T_{\text {eff }}$ are given in Table 5. The transit lightcurve is parameterised by the epoch of mid-transit $T_{0}$, the orbital period $P$, the planet-to-star area ratio $\left(R_{\mathrm{P}} / R_{*}\right)^{2}$, the approximate duration of the transit from initial to final contact $T_{14}$, and the impact parameter $b=a \cos i_{\mathrm{P}} / R_{*}$ (the distance, in fractional stellar radii, of the transit chord from the star's centre in the case of a circular orbit), where $a$ is the semimajor axis and $i_{\mathrm{P}}$ is the inclination of the orbital plane with respect to the sky plane.

The eccentric Keplerian RV orbit is parameterised by the stellar reflex velocity semi-amplitude $K_{1}$, the systemic velocity $\gamma$, an instrumental offset between the HARPS and CORALIE spectrographs $\Delta \gamma_{\text {HARPS }}$, and $\sqrt{e} \cos \omega$ and $\sqrt{e} \sin \omega$ where $e$ is orbital eccentricity and $\omega$ is the argument of periastron. We use $\sqrt{e} \cos \omega$ and $\sqrt{e} \sin \omega$ as they impose a uniform prior on $e$, whereas the jump parameters we used previously, $e \cos \omega$ and $e \sin \omega$, impose a linear prior that biases $e$ toward higher values (Anderson et al. 2011b). The RM effect was modelled using the formulation of Ohta et al. (2005) and, for similar reasons, is parameterised by $\sqrt{v \sin I} \cos \lambda$ and $\sqrt{v \sin I} \sin \lambda$.

The linear scale of the system depends on the orbital separation $a$ which, through Kepler's third law, depends on the stellar mass $M_{*}$. At each step in the Markov chain, the latest values of $\rho_{*}, T_{\text {eff }}$ and $[\mathrm{Fe} / \mathrm{H}]$ are input in to the empirical mass calibration of Enoch et al. (2010) (as based upon Torres et al. 2010, and as updated by Southworth 2011) to obtain $M_{*}$. The shapes of the transit lightcurves and the RV curve constrain stellar density $\rho_{*}$ (Seager \& Mallén-Ornelas 2003), which combines with $M_{*}$ to give the stellar radius $R_{*}$. The stellar effective temperature $T_{\text {eff }}$ and metallicity $[\mathrm{Fe} / \mathrm{H}]$ are proposal parameters constrained by Gaussian priors with mean values and variances derived directly from the stellar spectra (see Sect. 3).

As the planet-to-star area ratio is determined from the measured transit depth, the planet radius $R_{\mathrm{P}}$ follows from $R_{*}$. The planet mass $M_{\mathrm{P}}$ is calculated from the measured value of $K_{1}$ and the value of $M_{*}$; the planetary density $\rho_{\mathrm{P}}$ and surface gravity $\log g_{\mathrm{P}}$ then follow. We calculate the planetary equilibrium temperature $T_{\text {eql }}$, assuming zero albedo and efficient redistribution of heat from the planet's presumed permanent day side to its night side. We also calculate the durations of transit ingress $\left(T_{12}\right)$ and egress $\left(T_{34}\right)$.

At each step in the MCMC procedure, model transit lightcurves and RV curves are computed from the proposal parameter values, which are perturbed from the previous values by a small, random amount. The $\chi^{2}$ statistic is used to judge the goodness of fit of these models to the data and the decision as to whether to accept a step is made via the Metropolis-Hastings rule (Collier Cameron et al. 2007): a step is accepted if $\chi^{2}$ is lower than for the previous step and a step with higher $\chi^{2}$ is accepted with a probability proportional to $\exp \left(-\Delta \chi^{2} / 2\right)$. This gives the procedure some robustness against local minima and results in a thorough exploration of the parameter space around the best-fitting solution. To give proper weighting to each photometric data set, the uncertainties were scaled at the start of the MCMC so as to obtain a photometric reduced- $\chi^{2}$ of unity. To obtain a spectroscopic reduced- $\chi^{2}$ of unity we added "jitter" terms in quadrature to the formal RV errors of WASP-20: $12 \mathrm{~m} \mathrm{~s}^{-1}$ for the CORALIE RVs and $7 \mathrm{~m} \mathrm{~s}^{-1}$ for the HARPS RVs; no jitter was required for WASP-28. To account for e.g. a specific activity level, we partitioned the HARPS RVs of WASP-20 into two sets: those taken on the night of the spectroscopic transit observation of 2009 Oct. 22 and the remainder.

For both WASP-20b and WASP-28b we find, using the $F$-test approach of Lucy \& Sweeney (1971), that the improvement in the fit to the RV data resulting from the use of an eccentric orbit model is small and is consistent with the underlying orbit being circular. We thus adopt circular orbits, which Anderson et al. (2012) suggest is the prudent choice for short-period, Jupitermass planets in the absence of evidence to the contrary. We find $2 \sigma$ upper limits on $e$ of 0.11 and 0.14 for WASP-20b and WASP-28b, respectively.

Due to the low impact parameter of WASP-28b determinations of $v \sin I_{*}$ and $\lambda$ are degenerate (Triaud et al. 2011; Albrecht et al. 2011; Anderson et al. 2011a). To ensure $v \sin I_{*}$ is consistent with our spectroscopic measurement, we imposed a Gaussian prior on it by means of a Bayesian penalty on $\chi^{2}$, with mean and variance as determined from the HARPS spectra (see Sect. 3). From an MCMC run with no prior we obtained $\lambda=-8 \pm 54^{\circ}$ and $v \sin I_{*}=4.1_{-0.9}^{+5.9} \mathrm{~km} \mathrm{~s}^{-1}$, with $v \sin I_{*}$ reaching values as high as $30 \mathrm{~km} \mathrm{~s}^{-1}$, which is clearly inconsitent with the spectral measurement of $v \sin I_{*}=3.1 \pm 0.6 \mathrm{~km} \mathrm{~s}^{-1}$. The degeneracy between $v \sin I_{*}$ and $\lambda$ could be broken by line-profile tomography (Albrecht et al. 2007; Collier Cameron et al. 2010).

The median values and the $1 \sigma$ limits of our MCMC parameters' posterior distributions are given in Table 6 along with those of the derived parameters. The best fits to the RVs and the photometry are plotted in Figs. 1 and 2, with the RM effects highlighted in Fig. 3. There is no evidence of additional bodies in the RV residuals (Fig. 5). 
Table 5. Limb-darkening coefficients.

\begin{tabular}{llllllll}
\hline \hline Planet & Instrument & Observation bands & Claret band & $a_{1}$ & $a_{2}$ & $a_{3}$ & $a_{4}$ \\
\hline WASP-20 & WASP/EulerCam & Broad $(400-700 \mathrm{~nm}) / \mathrm{Gunn} r$ & Cousins $R$ & 0.578 & -0.048 & 0.493 & -0.294 \\
WASP-20 & FTN/FTS & Sloan $z^{\prime}$ & Sloan $z^{\prime}$ & 0.654 & -0.350 & 0.642 & -0.328 \\
WASP-28 & WASP/EulerCam & Broad (400-700 nm)/Gunn $r$ & Cousins $R$ & 0.428 & 0.456 & -0.175 & -0.023 \\
WASP-28 & FTN & Sloan $z^{\prime}$ & Sloan $z^{\prime}$ & 0.516 & 0.036 & 0.160 & -0.135 \\
\hline
\end{tabular}

Table 6. System parameters from the MCMC analyses.

\begin{tabular}{|c|c|c|c|c|}
\hline Parameter & Symbol & WASP-20 & WASP-28 & Unit \\
\hline Orbital period & $P$ & $4.8996284 \pm 0.0000033$ & $3.4088300 \pm 0.000006$ & $\mathrm{~d}$ \\
\hline Epoch of mid-transit & $T_{\mathrm{c}}$ & $2455715.65562 \pm 0.00029$ & $2455290.40519 \pm 0.00031$ & BJD (UTC) \\
\hline Transit duration & $T_{14}$ & $0.1418 \pm 0.0014$ & $0.1349 \pm 0.0010$ & $\mathrm{~d}$ \\
\hline Transit ingress/egress duration & $T_{12}=T_{34}$ & $0.0264 \pm 0.0016$ & $0.01441 \pm 0.00070$ & d \\
\hline Scaled orbital major semi-axis & $a / R_{*}$ & $9.28 \pm 0.23$ & $8.79 \pm 0.19$ & \\
\hline Planet-to-star area ratio & $\Delta F=R_{\mathrm{p}}^{2} / R_{*}^{2}$ & $0.01164 \pm 0.00023$ & $0.01300 \pm 0.00027$ & \\
\hline Impact parameter & $b$ & $0.718 \pm 0.018$ & $0.21 \pm 0.10$ & \\
\hline Orbital inclination & $i_{\mathrm{P}}$ & $85.56 \pm 0.22$ & $88.61 \pm 0.67$ & $\circ$ \\
\hline Stellar reflex velocity semi-amplitude & $K_{1}$ & $32.8 \pm 1.7$ & $120.5 \pm 4.2$ & $\mathrm{~m} \mathrm{~s}^{-1}$ \\
\hline Systemic velocity & $\gamma$ & $1316.300 \pm 0.089$ & $24216.41 \pm 0.43$ & $\mathrm{~m} \mathrm{~s}^{-1}$ \\
\hline Offset between HARPS \& CORALIE & $\Delta \gamma_{\text {HARPS }}$ & $19.3 \pm 1.3$ & $6.0 \pm 1.2$ & $\mathrm{~m} \mathrm{~s}^{-1}$ \\
\hline Offset between HARPS \& CORALIE & $\Delta \gamma_{\text {HARPS, orb }}$ & $13.31 \pm 0.16$ & - & $\mathrm{m} \mathrm{s}^{-1}$ \\
\hline Eccentricity & $e$ & 0 (adopted; $<0.11$ at $2 \sigma)$ & 0 (adopted; <0.14 at $2 \sigma$ ) & \\
\hline Sky-projected spin-orbit angle & $\lambda$ & $12.7 \pm 4.2$ & $8 \pm 18$ & $\circ$ \\
\hline Sky-projected stellar rotation velocity & $v \sin I_{*}$ & $4.75 \pm 0.51$ & $3.25 \pm 0.34$ & $\mathrm{~km} \mathrm{~s}^{-1}$ \\
\hline Stellar mass & $M_{*}$ & $1.200 \pm 0.041$ & $1.021 \pm 0.050$ & $M_{\odot}$ \\
\hline Stellar radius & $R_{*}^{*}$ & $1.392 \pm 0.044$ & $1.094 \pm 0.031$ & $R_{\odot}$ \\
\hline Stellar surface gravity & $\log g_{*}$ & $4.231 \pm 0.020$ & $4.370 \pm 0.018$ & (cgs) \\
\hline Stellar density & $\rho_{*}$ & $0.447 \pm 0.033$ & $0.784 \pm 0.058$ & $\rho_{\odot}$ \\
\hline Stellar effective temperature & $T_{\text {eff }}$ & $5940 \pm 100$ & $6150 \pm 140$ & $\mathrm{~K}$ \\
\hline Stellar metallicity & {$[\mathrm{Fe} / \mathrm{H}]$} & $-0.008 \pm 0.060$ & $-0.290 \pm 0.10$ & \\
\hline Planetary mass & $M_{\mathrm{P}}$ & $0.311 \pm 0.017$ & $0.907 \pm 0.043$ & $M_{\text {Jup }}$ \\
\hline Planetary radius & $R_{\mathrm{P}}$ & $1.462 \pm 0.059$ & $1.213 \pm 0.042$ & $R_{\text {Jup }}$ \\
\hline Planetary surface gravity & $\log g_{\mathrm{P}}$ & $2.530 \pm 0.036$ & $3.149 \pm 0.028$ & (cgs) \\
\hline Planetary density & $\rho_{\mathrm{P}}$ & $0.099 \pm 0.012$ & $0.508 \pm 0.047$ & $\rho_{\text {Jup }}$ \\
\hline Orbital major semi-axis & $a$ & $0.06000 \pm 0.00069$ & $0.04469 \pm 0.00076$ & AU \\
\hline Planetary equilibrium temperature & $T_{\mathrm{eql}}$ & $1379 \pm 31$ & $1468 \pm 37$ & $\mathrm{~K}$ \\
\hline
\end{tabular}
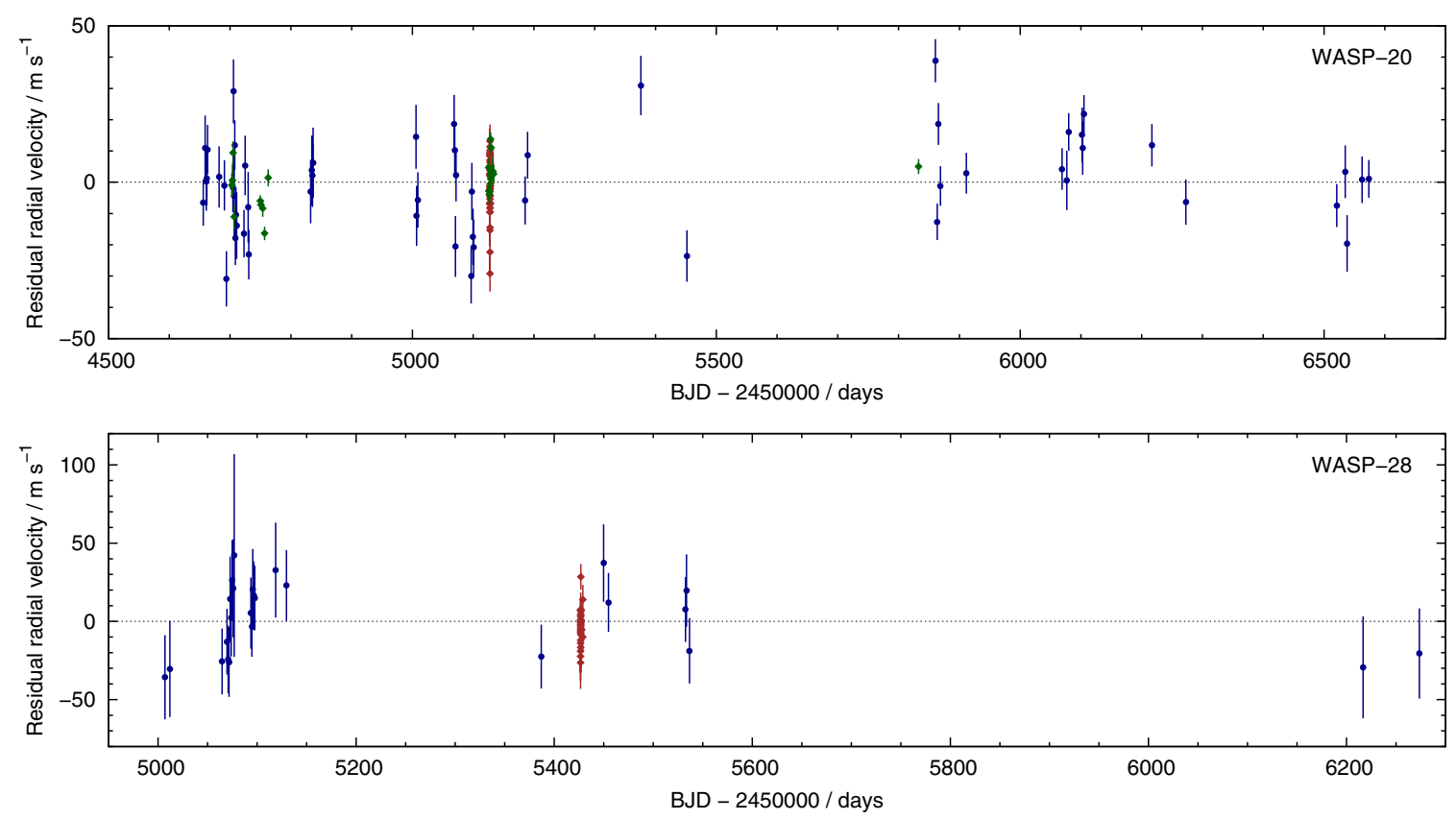

Fig. 5. Residuals about the best-fitting Keplerian orbits as a function of time (CORALIE in blue, HARPS in green, and HARPS transits in brown). There is no evidence of additional bodies. 

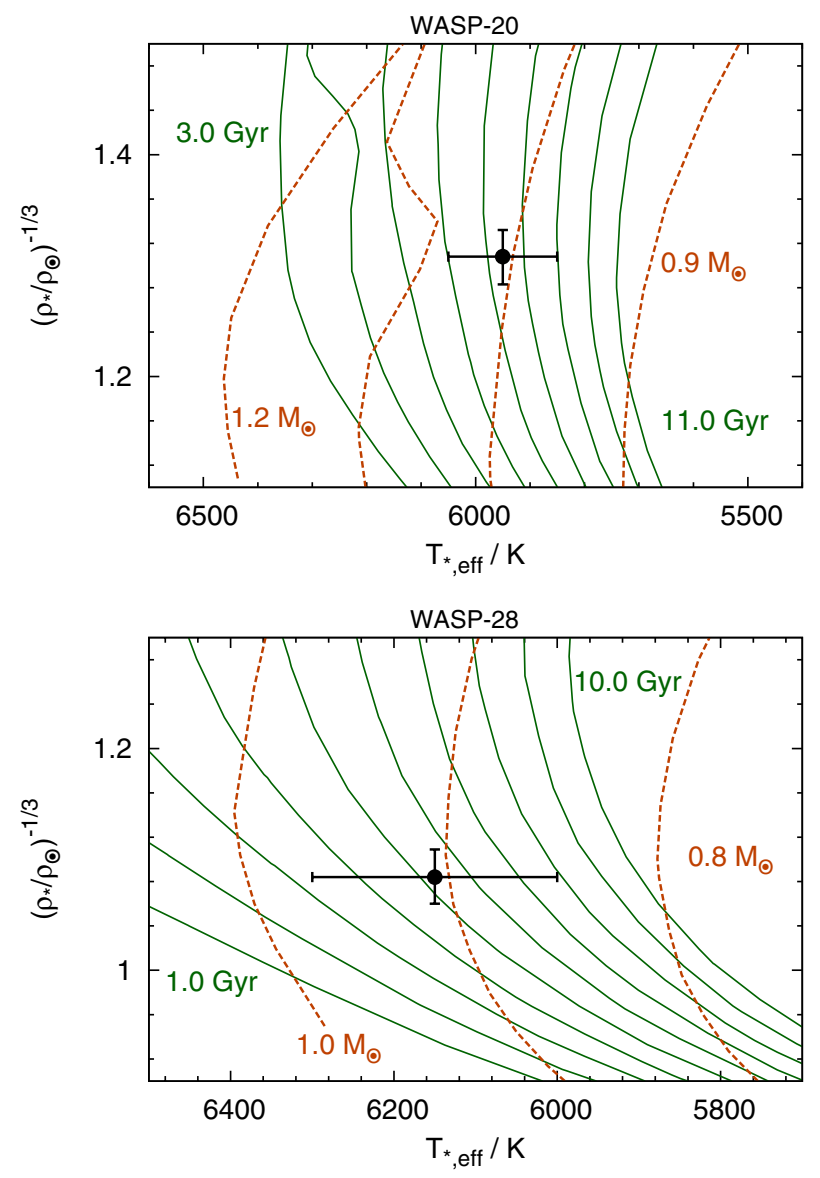

Fig. 6. Modified H-R diagrams. The isochrones, from Bressan et al. (2012), are in steps of integer Gyr with $Z=0.0152$ for WASP-20 and $Z=0.0078$ for WASP-28. The mass tracks, from Bertelli et al. (2008), are in steps of $0.1 M_{\odot}$ with $Z=0.019$ for WASP-20 and $Z=0.0097$ for WASP-28.

We interpolated the mass tracks of Bertelli et al. (2008) and the isochrones of Bressan et al. (2012) using $\rho_{*}, T_{\text {eff }}$ and $[\mathrm{Fe} / \mathrm{H}]$ from the MCMC analysis (Fig. 6). This suggests ages of $7_{-1}^{+2}$ Gyr for WASP-20 and $5_{-2}^{+3}$ Gyr for WASP-28 and masses of $1.02_{-0.03}^{+0.07} M_{\odot}$ for WASP-20 and $0.91 \pm 0.06 M_{\odot}$ for WASP-28. These are slightly lower than the masses derived from our MCMC analyses $\left(1.20 \pm 0.04 M_{\odot}\right.$ for WASP-20 and $1.02 \pm$ $0.05 M_{\odot}$ for WASP-28), though they are consistent at the $2.3 \sigma$ (WASP-20) and $1.4 \sigma$ (WASP-28) levels. This discrepancy between these mass estimates suggests that there may be additional factors not accounted for in the stellar models. It is not possible to quantify the additional uncertainty on the mass and age derived from the models without knowing the reason(s) for this discrepancy. It is also possible that these stars differ in some way from those used to establish the empirical mass calibration used in our MCMC analysis. This is certainly an interesting problem that warrants further investigation, but it is beyond the scope of this paper.

\section{Discussion}

We present the discovery of WASP-20b, a Saturn-mass planet in a 4.9-day orbit around CD-24 $102(V=10.7)$, and WASP-28b, a Jupiter-mass planet in a 3.4-day orbit around a $V=12$ star. Based on their masses, orbital distances, irradiation levels and metallicities, the radii of the planets $\left(1.46 R_{\text {Jup }}\right.$ for WASP-20b and $1.21 R_{\text {Jup }}$ for WASP-28b) are consistent with the predictions of the empirical relations of Enoch et al. (2012): $1.33 R_{\text {Jup }}$ for WASP-20b and $1.26 R_{\text {Jup }}$ for WASP-28b. Due to the low density of the planet and the apparent brightness of its host star, WASP-20 is a good target for atmospheric characterisation via transmission spectroscopy (e.g. Kreidberg et al. 2014).

We find both WASP-20b and WASP-28b to be in prograde orbits. The orbit of WASP-20b may be slightly misaligned with its star's spin: $\lambda=12.7 \pm 4.2^{\circ}$. Although our measurement for WASP-28b $\left(\lambda=8 \pm 18^{\circ}\right)$ is consistent with an aligned orbit, its low impact parameter results in a relatively large uncertainty. Using the period-colour relation for the Hyades of Delorme et al. (2011) and scaling to the inferred ages of the host stars using $P \propto t^{0.5}$, we predict rotation velocities consistent with the spectroscopic $v \sin I_{*}$ values: $v \sim 2.9 \mathrm{~km} \mathrm{~s}^{-1}$ for WASP-20 and $v \sim 2.1 \mathrm{~km} \mathrm{~s}^{-1}$ for WASP-28. This suggests that $I_{*} \approx 90^{\circ}$ and therefore that $\Psi \approx \lambda$.

Both host stars are near the posited boundary between efficient and inefficient aligners of $T_{\text {eff }} \approx 6250 \mathrm{~K}$ (Winn et al. 2010; Schlaufman 2010). Both systems, with their low obliquity determinations, are consistent with the observation that systems with cool host stars and short expected tidal timescales are aligned (Albrecht et al. 2012, and references therein). With $M_{\mathrm{P}} / M_{*}=0.00025$ and $a / R_{*}=9.3$, WASP-20b experiences relatively weak tidal forces. Its relative tidal dissipation timescale of $\tau_{\mathrm{CE}}=2.6 \times 10^{13} \mathrm{yr}$ is the longest for the cool-host group barring HD $17156 \mathrm{~b}$ (see Sect. 5.3 of Albrecht et al. 2012) ${ }^{2}$. With $M_{\mathrm{P}} / M_{*}=0.00084$ and $a / R_{*}=9.5, \tau_{\mathrm{CE}}=2.5 \times 10^{12} \mathrm{yr}$ for WASP-28b, placing it within the main grouping of aligned, coolhost systems. Assuming the planets did not arrive in their current orbits recently, the advanced age of both systems, $7_{-1}^{+2} \mathrm{Gyr}$ for WASP-20 and $5_{-2}^{+3}$ Gyr for WASP-28, mean tidal dissipation would have occurred over a long timescale.

The planets may have arrived in their current orbits via planet-disc migration (Lin et al. 1996; Marzari \& Nelson 2009) or via the scattering and circularisation route (Fabrycky \& Tremaine 2007; Nagasawa et al. 2008; Matsumura et al. 2010; Naoz et al. 2011). Assuming the latter, the planets may have scattered into near-aligned orbits or they may have aligned with the spins of their host stars via tidal interaction, though how this could have occurred without the planets falling into their stars is currently a mystery (Barker \& Ogilvie 2009). It will be interesting to discover if the small, significant misalignment of WASP-20 is confirmed by further observations and, perhaps, by a re-analysis of the existing data using the line-profile tomography technique (Albrecht et al. 2007; Collier Cameron et al. 2010). In that case the system may prove particularly useful in studies of the migration of hot Jupiters and the tidal damping of spin-orbit misalignments.

Acknowledgements. WASP-South is hosted by the South African Astronomical Observatory and SuperWASP-North is hosted by the Isaac Newton Group on La Palma. We are grateful for their ongoing support and assistance. Funding for WASP comes from consortium universities and from the UK's Science and Technology Facilities Council. M. Gillon is a FNRS Research Associate. A. H. M. J. Triaud is a Swiss National Science Foundation fellow under grant number PBGEP2-145594.

\section{References}

Albrecht, S., Reffert, S., Snellen, I., Quirrenbach, A., \& Mitchell, D. S. 2007, A\&A, 474, 565

Albrecht, S., Winn, J. N., Johnson, J. A., et al. 2011, ApJ, 738, 50

2 Note that these timescales are relative and that Albrecht et al. (2012) plot the timescales divided by $5 \times 10^{9}$. 
Albrecht, S., Winn, J. N., Johnson, J. A., et al. 2012, ApJ, 757, 18 Anderson, D. R., Collier Cameron, A., Gillon, M., et al. 2011a, A\&A, 534, A16 Anderson, D. R., Collier Cameron, A., Hellier, C., et al. 2011b, ApJ, 726, L19 Anderson, D. R., Collier Cameron, A., Gillon, M., et al. 2012, MNRAS, 422, 1988

Bakos, G., Noyes, R. W., Kovács, G., et al. 2004, PASP, 116, 266

Baranne, A., Queloz, D., Mayor, M., et al. 1996, A\&AS, 119, 373

Barker, A. J., \& Ogilvie, G. I. 2009, MNRAS, 395, 2268

Bate, M. R., Lodato, G., \& Pringle, J. E. 2010, MNRAS, 401, 1505

Bertelli, G., Girardi, L., Marigo, P., \& Nasi, E. 2008, A\&A, 484, 815

Borucki, W. J., Koch, D., Basri, G., et al. 2010, Science, 327, 977

Bressan, A., Marigo, P., Girardi, L., et al. 2012, MNRAS, 427, 127

Brown, T. M., Baliber, N., Bianco, F. B., et al. 2013, PASP, 125, 1031

Claret, A. 2000, A\&A, 363, 1081

Claret, A. 2004, A\&A, 428, 1001

Collier Cameron, A., Pollacco, D., Street, R. A., et al. 2006, MNRAS, 373, 799

Collier Cameron, A., Wilson, D. M., West, R. G., et al. 2007, MNRAS, 380, 1230

Collier Cameron, A., Bruce, V. A., Miller, G. R. M., Triaud, A. H. M. J., \& Queloz, D. 2010, MNRAS, 403, 151

Delorme, P., Collier Cameron, A., Hebb, L., et al. 2011, MNRAS, 413, 2218

Enoch, B., Collier Cameron, A., Parley, N. R., \& Hebb, L. 2010, A\&A, 516, A33

Enoch, B., Collier Cameron, A., \& Horne, K. 2012, A\&A, 540, A99

Fabrycky, D., \& Tremaine, S. 2007, ApJ, 669, 1298

Faedi, F., Barros, S. C. C., Pollacco, D., et al. 2011, Detection and Dynamics of Transiting Exoplanets, St. Michel l'Observatoire, France, eds. F. Bouchy, R. Díaz, \& C. Moutou, EPJ Web Conf., 11, 1003

Gray, D. F. 2008, The Observation and Analysis of Stellar Photospheres (Cambridge University Press)

Greaves, J. S., Kennedy, G. M., Thureau, N., et al. 2014, MNRAS, 438, L31

Hellier, C., Anderson, D. R., Collier Cameron, A., et al. 2011, Detection and Dynamics of Transiting Exoplanets, St. Michel l'Observatoire, France, eds. F. Bouchy, R. Díaz, \& C. Moutou, EPJ Web Conf., 11, 1004

Kreidberg, L., Bean, J. L., Désert, J.-M., et al. 2014, ApJ, 793, L27
Lai, D., Foucart, F., \& Lin, D. N. C. 2011, MNRAS, 412, 2790

Lendl, M., Anderson, D. R., Collier-Cameron, A., et al. 2012, A\&A, 544, A72

Lin, D. N. C., Bodenheimer, P., \& Richardson, D. C. 1996, Nature, 380, 606

Lucy, L. B., \& Sweeney, M. A. 1971, AJ, 76, 544

Magain, P. 1984, A\&A, 134, 189

Mandel, K., \& Agol, E. 2002, ApJ, 580, L171

Marzari, F., \& Nelson, A. F. 2009, ApJ, 705, 1575

Matsumura, S., Peale, S. J., \& Rasio, F. A. 2010, ApJ, 725, 1995

Maxted, P. F. L., Anderson, D. R., Collier Cameron, A., et al. 2011, PASP, 123, 547

Mayor, M., Pepe, F., Queloz, D., et al. 2003, The Messenger, 114, 20

McLaughlin, D. B. 1924, ApJ, 60, 22

Nagasawa, M., Ida, S., \& Bessho, T. 2008, ApJ, 678, 498

Naoz, S., Farr, W. M., Lithwick, Y., Rasio, F. A., \& Teyssandier, J. 2011, Nature, 473, 187

Ohta, Y., Taruya, A., \& Suto, Y. 2005, ApJ, 622, 1118

Pepe, F., Mayor, M., Queloz, D., et al. 2005, The Messenger, 120, 22 Pollacco, D. L., Skillen, I., Cameron, A. C., et al. 2006, PASP, 118, 1407 Pollacco, D., Skillen, I., Collier Cameron, A., et al. 2008, MNRAS, 385, 1576

Queloz, D., Mayor, M., Weber, L., et al. 2000, A\&A, 354, 99

Queloz, D., Henry, G. W., Sivan, J. P., et al. 2001, A\&A, 379, 279

Rogers, T. M., Lin, D. N. C., \& Lau, H. H. B. 2012, ApJ, 758, L6

Rossiter, R. A. 1924, ApJ, 60, 15

Schlaufman, K. C. 2010, ApJ, 719, 602

Seager, S., \& Mallén-Ornelas, G. 2003, ApJ, 585, 1038

Southworth, J. 2011, MNRAS, 417, 2166

Torres, G., Andersen, J., \& Giménez, A. 2010, A\&ARv, 18, 67

Triaud, A. H. M. J. 2011, A\&A, 534, L6

Triaud, A. H. M. J., Collier Cameron, A., Queloz, D., et al. 2010, A\&A, 524, A25

Triaud, A. H. M. J., Queloz, D., Hellier, C., et al. 2011, A\&A, 531, A24

Watson, C. A., Littlefair, S. P., Diamond, C., et al. 2011, MNRAS, 413, L71

Winn, J. N., Fabrycky, D., Albrecht, S., \& Johnson, J. A. 2010, ApJ, 718, L145

Zacharias, N., Monet, D. G., Levine, S. E., et al. 2004, BAAS, 36, 1418 